\title{
Impact of Training Modality on Strength and Physical Function in Older Adults
}

\author{
Mark M. Misic $^{a}$ Rudy J. Valentine ${ }^{b}$ Karl S. Rosengren ${ }^{c}$ Jeffrey A. Woods ${ }^{b}$ \\ Ellen M. Evans ${ }^{b}$ \\ ${ }^{a}$ Department of Kinesiology and Physical Education, Northern Illinois University, DeKalb, III., b Department of \\ Kinesiology and Community Health, University of Illinois at Urbana-Champaign, Urbana, III., and 'Department of \\ Psychology, Northwestern University, Evanston, III., USA
}

\section{Key Words \\ Leg extension and flexion · Cardiovascular training • Flexibility training $\cdot$ Lower extremity physical function}

\begin{abstract}
Background: The importance of maintaining strength in older adults is well documented and various training modalities have been recommended; however, the effectiveness of various interventions with high translation to public health practice has not been completely characterized. Additionally, the interrelations among mode, strength, and lower extremity physical function (LEPF) changes in older adults warrant further investigation. Purpose: The primary aims were to examine the effectiveness of cardiovascular endurance training (CVE) compared with balance and flexibility training (FLEX) and to explore the relationship of muscle strength to LEPF. Methods: Fifty-five adults (69.1 \pm 5.6 years, 35 female) were randomly assigned to 10 months of CVE or FLEX. Knee extension and flexion at 60 and $120^{\circ} \cdot \mathrm{s}^{-1}$ were measured using an isokinetic dynamometer. LEPF was measured using a battery of tasks. The CVE group exercised using treadmills, cycles, and elliptical trainers. The FLEX group participated in a group exercise class that incorporated flexibility and balance activities. Results: The CVE training group significantly improved peak oxygen consumption by $6 \%$ $(p=0.03)$, while the FLEX training group showed no change $(p=0.47)$. Participants significantly improved peak torque at both speeds and muscle actions by $21-65 \%$ ( $\leq \leq 0.05$ ), with
\end{abstract}

(C) 2009 S. Karger AG, Basel

0304-324X/09/0554-0411\$26.00/0

Fax +41613061234

E-Mail karger@karger.ch

www.karger.com
Accessible online at:

www.karger.com/ger no group effect ( $p>0.05)$. Both groups also significantly improved performance on all LEPF tasks included in this study $(4-7 \%, p \leq 0.05)$. Significant correlations (all $p=0.00$ ) were found between improvements in strength and improvements in LEPF including timed walk and peak torque extension and flexion at $60^{\circ} \cdot \mathrm{s}^{-1}(r=0.40$ and 0.27 , respectively), obstacle walk and peak torque extension and flexion at 60 and $120^{\circ} \cdot \mathrm{s}^{-1}$ ( $\mathrm{r}$ range $0.27-0.40$ ), and stair ascent and peak torque extension at $120^{\circ} \cdot \mathrm{s}^{-1}(\mathrm{r}=0.37)$. Conclusions: Sedentary older adults achieve similar improvements in strength and LEPF with either CVE or FLEX training with the latter being related to improvements in leg strength.

Copyright $\odot 2009$ S. Karger AG, Base

The preservation of physical function is a high priority for older adults in order to maintain independent living [1]. A key aspect of physical function is lower extremity strength which has been documented as a significant predictor of falling risk in older adults [2] and is related to increased difficulty in performing activities of daily living [3]. Epidemiologic data support the relationship between physical activity, strength development or maintenance, and reductions in fall incidence [4]. Even beyond reducing the risk of falling, preservation of strength has been shown to be related to mortality [5].

A variety of training programs and modalities have been recommended to maintain and improve strength in older adults. These include traditional strength training 
modalities such as free weights [6], machines [7], and other devices such as bands [8], or using specific exercise training methods such as Taiji [9], Pilates, or yoga [10]. What has been poorly characterized, however, is the impact that commonly prescribed exercise modes, not explicitly strength training, may have on lower extremity strength. The exercise regimens have ranged from cardiovascular training to flexibility and/or balance programs which have been prescribed for other health benefits of importance to the older population [11]. Moreover, longer-duration training interventions (i.e. longer than 6 months) have been understudied.

The specificity principle of training establishes that if the goal is to improve leg strength, then leg strength training should have the greatest impact. However, when working with an older population, considering issues with long-term adherence to a training program and additional health goals are important. Therefore, the manner in which strength training may be achieved and sustained is of interest and alternative approaches beyond conventional strength training need to be considered. Additionally, the relationship between fitness improvements and functional measures of physical performance in the elderly is unclear $[3,12,13]$.

In this context, the current study had two primary aims: (1) to determine the relative efficacy of cardiovascular training compared with balance and flexibility training to increase lower extremity strength in older adults, and (2) to determine the relation between changes in fitness, including muscular strength and aerobic capacity, and lower extremity physical function (LEPF) in older adults.

\section{Methods}

\section{Subjects}

Fifty-five healthy community-dwelling older adults (20 men, 35 women, aged $69.1 \pm 5.6$ years, range $60-85$ years) were recruited for participation in the present study from a parent study designed to assess the impact of cardiovascular exercise training on immune function. To be eligible for participation, participants needed to be relatively sedentary for a minimum of 6 months prior to starting the study (defined as no structured exercise program), have a body mass index (BMI) between 22 and 38, and have received medical clearance from their primary physician. Individuals with uncontrolled cardiovascular or metabolic disease, cancer, severe respiratory conditions, HIV or orthopedic limitations that would prevent them from completing the testing and training were not eligible for participation. All procedures used in the study were approved by the Institutional Review Board and all subjects signed an informed consent document prior to enrollment in the study. Once accepted into the study, the subjects were randomly assigned to a cardiovascular endurance (CVE) training group ( $\mathrm{n}=26 ; 17$ women) or a flexibility and balance training group (FLEX: $\mathrm{n}=29 ; 18$ women).

\section{Assessments}

Aerobic Fitness. A graded treadmill exercise test was conducted to determine maximal aerobic capacity and heart rate, blood pressure, and ECG responses to exercise. Oxygen uptake was measured continuously and recorded every $30 \mathrm{~s}$ using open-circuit spirometry (Parvomedics True Max 2400, Sandy, Utah, USA). Subjects walked on a treadmill at the fastest comfortable pace at $0 \%$ grade for 3-4 min, after which the grade increased by $1-2 \%$ every 1 or $2 \mathrm{~min}$. The test continued until the subjects were unable to continue due to volitional exhaustion, ECG changes or other abnormalities presented that rendered it unsafe to continue to exercise. Subjects exhibiting ECG changes or abnormalities were excluded from the study and referred to their primary physician. Maximal aerobic capacity is conventionally determined by the highest oxygen uptake that corresponds to at least 2 of the following criteria: (a) a plateau in aerobic capacity values despite an increase in exercise intensity, (b) maximal heart rate within 10 beats/min of the age-predicted maximum (220 beats/min minus age in years), and (c) a respiratory exchange ratio greater than 1.10. Because most but not all participants attained a true maximal value, peak aerobic capacity was determined as the highest attained oxygen consumption and was operationally defined as $\mathrm{VO}_{2 \text { peak. }}$. Those participants who failed to meet the criteria for maximal capacity most often cited shortness of breath and fatigue as the reasons for terminating the test.

Muscular Strength. Muscular strength was assessed using an isokinetic dynamometer (Biodex System 3, New York, N.Y., USA). Bilateral reciprocal concentric contractions at the knee were measured at preset velocities of $60^{\circ} \cdot \mathrm{s}^{-1}$ and $120^{\circ} \cdot \mathrm{s}^{-1}$. This joint was selected due to past research identifying weak lower extremities as potential factors leading to falls $[14,15]$. These speeds were selected to test a lower and upper speed of movement and were also based on previous research that investigated the relationship of strength to LEPF in older adults $[12,16]$. A familiarization trial was performed by each subject at both velocities and then, following a rest interval to allow full recovery, two trials at each speed were performed. Approximately 2 min of rest were allowed between trials. The highest peak torque extension (EXT) and peak torque flexion (FLX) achieved at $60^{\circ} \cdot \mathrm{s}^{-1}$ and $120^{\circ} \cdot \mathrm{s}^{-1}$ for each contraction were recorded and provided four distinct measures of muscular strength (EXT60, FLX60, EXT120, FLX120).

Lower Extremity Physical Function. The Berg Balance Scale [17] was used to screen potential participants for a major balance disability that would preclude safely undergoing the full battery of testing using a cut-off score of 45 (out of a possible score of 54). Gait performance was assessed using a timed up-and-go (UPGO) task, a 7-meter walk (WALK) and a 7-meter walk with obstacle (OBWALK). For the UPGO, subjects sat in a chair and on the command 'go' were told to stand and move as quickly as possible around a cone placed $2.5 \mathrm{~m}$ away from the chair, return to the chair and sit down while being timed. Similarly, each subject completed two trials of a 7-meter walk with and without stepping over a $30-\mathrm{cm}$ obstacle at the 4-meter point, walking at their normal pace. Subjects also performed a stair ascent (STAIRUP) and descent (STAIRDOWN) on a flight of 15 stairs (18-cm riser, $27-\mathrm{cm}$ tread) and were instructed to ascend and descend at their normal 
pace, preferably without using the handrail if they could do so safely. The handrail was only used to assist with balance and could not be used to assist climbing up the stairs.

Exercise Training. Subjects in the CVE group exercised 3 days a week for approximately $45 \mathrm{~min} /$ day for 10 months. Exercise sessions were completed on traditional cardiovascular machines including treadmills, stationary cycles, elliptical machines, and stair stepping machines. The target intensity was defined as $75 \%$ of heart rate reserve maximum $\left(H R R_{\max }\right)$, based on the subject's initial aerobic fitness test. Subjects exercised individually, but the exercise sessions took place during established times where 6-12 subjects were exercising simultaneously. CVE subjects wore Polar heart rate monitor transmission strap (Polar, Finland) during all exercise sessions. Intensity was increased progressively with the goal being for each subject to be exercising at $75 \%$ of $H R R_{\max }$ within 2 months of beginning the exercise intervention. FLEX subjects exercised in a group fitness setting 2 days each week for approximately $60 \mathrm{~min} /$ day for 10 months. Each class included stretching and a wide variety of activities ranging from balance training using half foam rollers, stability balls, BOSU ${ }^{\circledR}$ domes, agility ladders, and hurdles, to functional exercises incorporating medicine balls, resistance bands, and dumbbells. Due to restrictions imposed by the parent study design, the FLEX classes were kept at a low aerobic intensity where heart rates did not exceed approximately $33 \%$ of $\mathrm{HRR}_{\max }$. FLEX subjects' heart rates were regularly checked during the exercise intervention to ensure they did not exceed the target intensities. FLEX classes rotated through all types of training and were not designed to be progressive in nature. Exercises focused on performing movements through a full range of motion with repetitions ranging from 8 to 15 .

\section{Statistical Analyses}

All data analyses were conducted using SPSS version 16.0 (SPSS Inc., Chicago, Ill., USA). Means, standard deviations, and distribution statistics (skewness and kurtosis) were calculated to ensure that assumptions of normality were met for subsequent analyses. An intention-to-treat analysis was followed and in those cases where postintervention tests were not completed, the last observation from the pretesting was carried forward. The primary analysis used a repeated-measures (time by group) ANOVA to determine relative effectiveness of the interventions on outcomes of interest. To determine the effectiveness of each respective intervention, paired sample $t$ tests were used for comparing the baseline and posttest scores. To explore the relation between changes in strength outcomes and aerobic fitness and LEPF outcomes, correlation coefficients were assessed. Data are expressed as means and standard deviations in text and tables. All significance tests were conducted at the $\mathrm{p} \leq 0.05$ level.

\section{Results}

Four women ( 3 in CVE) and 4 men ( 2 in CVE) were unable to complete all postintervention assessments. Seven of these cases were due to medical conditions independent from the training intervention including cardiovascular-related disease, mobility impairments, or surgery. One subject was unable to complete posttesting due to

Training Modality Impact on Strength and Function Change
Table 1. Baseline subject characteristics

\begin{tabular}{lccc}
\hline Characteristic & $\begin{array}{l}\text { FLEX } \\
(\mathrm{n}=29)\end{array}$ & $\begin{array}{l}\text { CVE } \\
(\mathrm{n}=26)\end{array}$ & $\begin{array}{l}\text { Total } \\
(\mathrm{n}=55)\end{array}$ \\
\hline Age, years & $70.24 \pm 6.70$ & $67.85 \pm 3.91$ & $69.11 \pm 5.63$ \\
Weight, kg & $79.57 \pm 14.56$ & $76.68 \pm 15.92$ & $78.20 \pm 15.14$ \\
Height, m & $1.65 \pm 0.10$ & $1.66 \pm 0.10$ & $1.65 \pm 0.10$ \\
BMI & $29.13 \pm 3.46$ & $27.92 \pm 5.05$ & $28.56 \pm 4.29$ \\
Body fat, \% & $35.69 \pm 6.94$ & $34.78 \pm 8.02$ & $35.26 \pm 7.41$ \\
$\mathrm{VO}_{2 \text { peak }}$ & & & \\
$\quad$ ml/kg/min & $19.22 \pm 4.56$ & $21.10 \pm 4.70$ & $20.11 \pm 4.69$ \\
EXT60, Nm & $108.27 \pm 47.21$ & $108.57 \pm 47.30$ & $108.41 \pm 46.81$ \\
FLX60, Nm & $45.90 \pm 23.11$ & $49.77 \pm 21.97$ & $47.73 \pm 22.45$ \\
EXT120, Nm & $89.96 \pm 40.01$ & $87.13 \pm 40.33$ & $88.62 \pm 39.81$ \\
FLX120, Nm & $39.92 \pm 20.58$ & $44.06 \pm 25.15$ & $41.88 \pm 22.73$ \\
\hline
\end{tabular}

No significant differences existed on any variable between groups.

work conflict. Participation compliance for the two groups was $81 \%$ for the FLEX and $74 \%$ for the CVE for the 10-month training intervention. Subject characteristics are summarized in table 1 . Baseline strength measurements of peak torque extension and flexion were equal in the two training groups. All subjects improved peak torque at the knee for flexion and extension for both speeds tested with no significant differences in torque improvement between the two training modalities (table 2). As expected, the CVE training group significantly improved $\mathrm{VO}_{2 \text { peak }}$ by an average of $6 \%(\mathrm{p}=0.03)$, while the FLEX training group showed no statistically significant change $(0 \%, \mathrm{p}=0.47)$.

All LEPF measures improved by the end of the training period within both training groups ( $p$ range of 0.00 to 0.03 ), but there were no significant differences in improvement between the two training modalities (table 2, all $\mathrm{p}>0.05)$. Table 3 shows the correlations between the improvement in strength, improvement in $\mathrm{VO}_{2 \text { peak }}$, and change in LEPF performance. Significant correlations (all $\mathrm{p}=0.00$ ) were found between improvements in strength and improvements in LEPF. Specifically, the improvement in WALK was significantly related to the improvement in EXT60 and FLX60 ( $r=0.40$ and 0.27). The improvement in OBWALK was significantly related to the improvement in EXT60 and FLX60 ( $\mathrm{r}=0.40$ and 0.29$)$ and EXT120 and FLX120 ( $r=0.28$ and 0.27). Additionally, the improvement in STAIRUP was significantly related to improvement in EXT120 ( $\mathrm{r}=0.37$ ). No significant relations were found between improvement in any LEPF test and improvement in $\mathrm{VO}_{2 \text { peak }}$ performance $(\mathrm{p}>0.05)$. 
Table 2. Changes in performance in response to the interventions

\begin{tabular}{|c|c|c|c|}
\hline & $\begin{array}{l}\text { FLEX } \\
(\mathrm{n}=29)\end{array}$ & $\begin{array}{l}\text { CVE } \\
(n=26)\end{array}$ & $\begin{array}{l}\text { Total } \\
(\mathrm{n}=55)\end{array}$ \\
\hline \multirow[t]{2}{*}{ EXT60, Nm } & $21.08 \pm 25.94^{\mathrm{a}}$ & $14.14 \pm 17.41^{\mathrm{a}}$ & $17.80 \pm 22.39$ \\
\hline & $30 \%$ & $21 \%$ & $26 \%$ \\
\hline \multirow[t]{2}{*}{ FLX60, Nm } & $11.21 \pm 15.28^{\mathrm{a}}$ & $12.35 \pm 23.45^{\mathrm{a}}$ & $11.75 \pm 19.39$ \\
\hline & $65 \%$ & $36 \%$ & $51 \%$ \\
\hline \multirow[t]{2}{*}{ EXT120, Nm } & $15.49 \pm 15.13^{\mathrm{a}}$ & $10.62 \pm 24.00^{\mathrm{a}}$ & $13.19 \pm 19.78$ \\
\hline & $29 \%$ & $31 \%$ & $30 \%$ \\
\hline \multirow[t]{2}{*}{ FLX120, Nm } & $10.55 \pm 12.41^{\mathrm{a}}$ & $8.15 \pm 13.10^{\mathrm{a}}$ & $9.41 \pm 12.68$ \\
\hline & $57 \%$ & $42 \%$ & $50 \%$ \\
\hline \multirow{2}{*}{$\begin{array}{l}\mathrm{VO}_{2 \text { peak }} \\
\quad \mathrm{ml} / \mathrm{kg} / \mathrm{min}^{\mathrm{b}}\end{array}$} & $-0.37 \pm 2.71$ & $1.20 \pm 2.50^{\mathrm{a}}$ & $0.37 \pm 2.71$ \\
\hline & $0 \%$ & $+6 \%$ & $3 \%$ \\
\hline \multirow[t]{2}{*}{ STAIRUP, s } & $-0.65 \pm 1.92^{\mathrm{a}}$ & $-0.68 \pm 0.80^{\mathrm{a}}$ & $-0.66 \pm 1.49$ \\
\hline & $-6 \%$ & $-8 \%$ & $-7 \%$ \\
\hline \multirow[t]{2}{*}{ STAIRDOWN, s } & $-0.27 \pm 1.48^{\mathrm{a}}$ & $-0.71 \pm 1.06^{\mathrm{a}}$ & $-0.48 \pm 1.31$ \\
\hline & $-4 \%$ & $-8 \%$ & $-6 \%$ \\
\hline \multirow[t]{2}{*}{ WALK, m/s } & $0.04 \pm 0.13^{\mathrm{a}}$ & $0.05 \pm 0.11^{\mathrm{a}}$ & $0.05 \pm 0.12$ \\
\hline & $4 \%$ & $5 \%$ & $5 \%$ \\
\hline \multirow[t]{2}{*}{ OBWALK, m/s } & $0.04 \pm 0.11^{\mathrm{a}}$ & $0.05 \pm 0.10^{\mathrm{a}}$ & $0.04 \pm 0.11$ \\
\hline & $4 \%$ & $5 \%$ & $4 \%$ \\
\hline \multirow[t]{2}{*}{$\mathrm{UPGO}, \mathrm{m} / \mathrm{s}$} & $0.10 \pm 0.27^{\mathrm{a}}$ & $0.12 \pm 0.45^{\mathrm{a}}$ & $0.11 \pm 0.36$ \\
\hline & $4 \%$ & $6 \%$ & $5 \%$ \\
\hline
\end{tabular}

Decrease in STAIRUP and STAIRDOWN time represents improvement while increase in WALK, OBWALK, and UPGO speed represents improvement.

a Indicates significant within group change $(\mathrm{p}=0.00)$.

${ }^{\mathrm{b}}$ Indicates significant group by time change $(\mathrm{p}=0.03)$.

\section{Discussion}

The results from this study reinforce the value of physical activity in older adults and extend the current research in this area by considering the dynamic relation of training specificity to changes in muscular and aerobic fitness and to subsequent changes in lower extremity physical function. Most notable of the findings from the current study is that lower extremity strength improved in older adults equally, regardless of the training modality used. That is, older adults who trained using traditional cardiovascular equipment such as treadmills, stationary cycles, or elliptical machines improved in lower extremity strength measures equivalent to older adults who engaged in flexibility and balance training at low (cardiovascular) intensities. The improvements overall in strength ranged from 26 to $51 \%$ compared with baseline measurements. This finding underscores that strength improves if older adults engage in regular exercise, and the improvements are similar whether this exercise is
Table 3. Pearson correlation coefficients between improvement in fitness and improvement in LEPF tasks

\begin{tabular}{|c|c|c|c|c|c|}
\hline & \multicolumn{4}{|c|}{$\begin{array}{l}\text { Improvement in peak torque extension } \\
\text { and flexion at selected velocities }\end{array}$} & \multirow{2}{*}{$\begin{array}{l}\text { Improve- } \\
\text { ment in } \\
\mathrm{VO}_{2 \text { peak }}\end{array}$} \\
\hline & EXT60 & FLX60 & EXT120 & FLX120 & \\
\hline STAIRUP & 0.23 & 0.16 & $0.37^{*}$ & 0.15 & 0.02 \\
\hline STAIRDOWN & 0.16 & 0.10 & 0.24 & -0.03 & 0.14 \\
\hline WALK & $0.40^{*}$ & $0.27^{*}$ & 0.26 & 0.24 & 0.12 \\
\hline OBWALK & $0.40^{*}$ & $0.29 *$ & $0.28^{*}$ & $0.27^{*}$ & 0.22 \\
\hline UPGO & -0.12 & 0.15 & -0.04 & 0.09 & -0.15 \\
\hline
\end{tabular}

${ }^{*} \mathrm{p}=0.00$, significant correlation.

specifically cardiovascular or balance/flexibility training. The exception noted in this study and well documented in the literature was that if the goal was to improve cardiovascular fitness, as defined by peak oxygen consumption, this positive adaptation was only seen in those individuals who performed cardiovascular training.

Comparing these results with other studies is difficult given the novel focus of this study. However, other research which investigated the improvement in fitness across a wide variety of exercise modes also noted that often aerobic training and flexibility training resulted in comparable improvements in strength. Takeshima et al. [13] found no difference in improvement in muscular endurance between aerobic-trained older adults and flexibility-trained adults, although they only measured lower body streng th changes using an indirect measure of muscular endurance (a functional chair stand test). Notably, however, the current study differed from that of Takeshima et al. [13] and other similar studies with respect to the duration of the training intervention. The current study used a 10-month intervention, while the study of Takeshima et al. [13] was only 12 weeks in duration.

Iwamoto et al. [18] evaluated the efficacy of balance and strength training using a single group design in a slightly older sample (average age $=72.8$ years) compared with the current study (average age $=69.1$ years) with all subjects participating in both balance and strength training. They found maximal knee extension torque improved by only $6.8 \%$ in their subjects (compared with an average $26-30 \%$ improvement in the current study). The form of training in this study included similar elements to the current study (balance exercises, body weight squats), but these were done on a daily basis with each session much shorter than in the current study. Addi- 
tionally, the intervention was only 3 months in duration compared with the 10 months of the present study.

A more aggressive training program was implemented by Galvao and Taaffe [7] in older adults comparable in age to the subjects in the current study. Their program used traditional resistance training machines and included either a 1-set or 3-set protocol that at the end of 20 weeks of twice a week training resulted in a 20 (1-set training) to $24 \%$ (3-set training) improvement in peak knee extension torque. These improvements are comparable to the current study; however, the subjects in the Galvao and Taaffe study followed a training program designed around strength training rather than the broader and less specific training used in the current study. This distinction underscores the unique finding in the current study that older adults need not perform only strength training in order to experience significant strength improvements.

Christou et al. [9] investigated the impact of Taiji training on knee extensor force. Although they measured only one leg (the nondominant leg) and used an isometric contraction, they found a significant improvement in force of $20 \%$ after 20 weeks of $3 \mathrm{~h}$ /week Taiji training and no change in the control group. Again, their work reinforces that older adults can gain strength improvements from a nonresistance training form of training as the current study demonstrated.

Improvements in leg strength are of limited value if there is not a corresponding improvement in physical function. Although weakness and/or reduced leg strength is cited as being linked to disability [19], frailty [20], and fall risk [2, 21, 22], the connection between improved leg strength and improved LEPF is less well characterized. Based on the measures of LEPF used in this study, older adults improved an overall average of $4-7 \%$ in the physical functional tests used in this study regardless of the training modality.

The relationship between improvements in strength and improvement in LEPF was further assessed in this study, and the results demonstrated that improvement in leg extension peak torque had the strongest relationship to most changes in LEPF. This relationship was stronger than the relationship to changes in cardiovascular fitness. As suggested by the literature, improving the ability to perform activities of daily living appears more closely linked to improving muscular fitness compared with cardiovascular fitness. For example, Takeshima et al. [13], who also found that a variety of training modes resulted in improvements in strength, investigated changes in performance for each training mode. Similar to the cur-

Training Modality Impact on Strength and Function Change rent study, they also found a significant improvement in performance in the UPGO test for all training modes with the exception being their group that performed only balance exercises. The balance subjects outperformed aerobic- and flexibility-trained subjects in the chair stand as well as the UPGO, which the researchers attributed to greater muscle activation due to extensive training on one leg and/or on unstable surfaces.

It should be noted in the present study that the correlation coefficients between improvements in LEPF and improvements in strength were small to moderate ( $\mathrm{r}$ range 0.03 to 0.40 ). This underscores the contribution of other changes occurring in response to training beyond leg strength improvement, which could include psychological changes such as self-efficacy and-confidence [23], reaction time [24], or an interaction of factors related to movement and mobility.

The current study is not without its own limitations. Only bilateral leg extension and flexion were measured using preset velocities of 60 and $120^{\circ} \cdot \mathrm{s}^{-1}$. Other researchers have used different velocities, although no consensus exists in the literature as to the most predictive speed as it relates to fall prevention or functional performance in older adults. Furthermore, researchers have suggested ankle stability as a contributing factor to lower extremity performance and fall risk [25]; however, ankle performance was not measured in the current study. Additionally, no control group was used in this study, although its absence has limited if any implications for the conclusions of the study since a spontaneous improvement in strength and physical function is highly unlikely in the absence of exercise in this population. Furthermore, as the goal of this study was to determine the relative effectiveness of two modalities, a parallel-arm design is appropriate.

Although some of the performance measures only showed a small change as a result of the intervention, the sample of older adults recruited and trained in this study may also have represented higher functioning adults given that they were community-dwelling adults who lived on their own and had to be robust enough to exercise 2-3 days per week. Other researchers who found similar results to the current study where alternative training forms and lower intensity of training were used speculated that nondisabled and nonfrail older adults may already be near their performance ceiling on certain tasks and therefore improvement is unlikely [7]. Additionally, as Galvao and Taaffe [7] point out, despite the lack of improvement in LEPF, an improvement in strength may provide older adults with an added reserve capacity that places them 
above the level required to maintain independence and carry out tasks associated with daily living. Lastly, our results do not allow a distinction between statistical and clinical significance in terms of how changes in physical function noted in the current study translate into changes in performing activities of daily living.

In summary, the current study suggests that previously sedentary older adults may achieve comparable improvements in leg strength and LEPF through either CVE training or flexibility and balance training. The improvements in leg strength may explain some of the improvements in physical function, but undoubtedly other fac- tors also contribute to these improvements. Further research is warranted to determine exercise programs that are efficacious in terms of preserving physical function in older adults, but that also have high translational potential in terms of resources and adherence.

\section{Acknowledgements}

This study was supported by NIH/NIA AG18861 (J.A.W.) and University of Illinois Urbana-Champaign Research Board (E.M.E.).

\section{References}

1 Tannenbaum C, Mayo N: Women's health priorities and perceptions of care: a survey to identify opportunities for improving preventative health care delivery for older women. Age Ageing 2003;32:626-635.

$\checkmark 2$ Moreland JD, Richardson JA, Goldsmith $\mathrm{CH}$, Clase CM: Muscle weakness and falls in older adults: a systematic review and metaanalysis. J Am Geriatr Soc 2004;52:11211129.

3 Landers KA, Hunter GR, Wetzstein CJ, Bamman MM, Weinsier RL: The interrelationship among muscle mass, strength, and the ability to perform physical tasks of daily living in younger and older women. J Gerontol Biol Sci 2001;6A:B443-B448.

4 Buchner DM: Preserving mobility in older adults. West J Med 1997;167:258-264.

5 Newman AB, Kupelian V, Visser M, Simonsick EM, Goodpaster BH, Kritchevsky SB, Tylavsky FA, Rubin SM, Harris BH: Strength, but not muscle mass, is associated with mortality in the Health, Aging and Body Composition study cohort. J Gerontol Med Sci 2006;61A:72-77.

-6 Brill PA, Probst JC, Greenhouse DL, Schell B, Macera CA: Clinical feasibility of a freeweight strength-training program for older adults. J Am Board Fam Pract 1998;11:445451.

7 Galvao DA, Taaffe DR: Resistance exercise dosage in older adults: single- versus multiset effects on physical performance and body composition. J Am Geriatr Soc 2005;53: 2090-2097.

$\checkmark 8$ Manor B, Topp R, Page P: Validity and reliability of measurements of elbow flexion strength obtained from older adults using elastic bands. J Geriatr Phys Ther 2006;29: 18-21.

9 Christou EA, Yang Y, Rosengren KS: Taiji training improves knee extensor strength and force control in older adults. J Gerontol Med Sci 2003;58A:763-766.
10 Hill K, Smith R, Fearn M, Rydberg M, Oliphant R: Physical and psychological outcomes of a supported physical activity program for older carers. J Aging Phys Act 2007;15:257-271.

11 Cress ME, Buchner DM, Prohaska T, Rimmer J, Brown M, Macera C, Dipietro L, Chodzko-Zajko W: Best practices for physical activity programs and behavior counseling in older adult populations. J Aging Phys Act 2005; 13:61-74.

12 Carmeli E, Reznick AZ, Coleman R, Carmeli $\mathrm{V}$ : Muscle strength and mass of lower extremities in relation to functional abilities in elderly adults. Gerontology 2000;46:249-257.

13 Takeshima N, Rogers NL, Rogers ME, Islam MM, Koizumi D, Lee S: Functional fitness gain varies in older adults depending on exercise mode. Med Sci Sports Exerc 2007;39: 2036-2043.

14 Zizza CA, Herring A, Stevens J, Popkin BM: Obesity affects nursing-care facility admission among whites but not blacks. Obes Res 2002;10:816-823.

15 Krahnstoever K, Ford ES, Cogswell ME, Dietz WH: Percentage of body fat and body mass index are associated with mobility limitations in people aged 70 and older from NHANES III. J Am Geriatr Soc 2002;50: 1802-1809.

16 Jubrias SA, Odderson IR, Esselman PC, Conley KE: Decline in isokinetic force with age: muscle cross-sectional area and specific force. Eur J Physiol 1991;434:246-253.

17 Berg K, Wood-Dauphinee S, Williams JI, Maki, B: Measuring balance in the elderly: validation of an instrument. Can J Pub Health 1992;83(suppl 2):S7-S11.

- 18 Iwamoto J, Otaka Y, Kudo K, Takeda T, Uzawa M, Hirabayashi K: Efficacy of training program for ambulatory competence in elderly women. Keio J Med 2004;53:85-89.
19 Guralnik JM, Ferrucci L, Pieper CF, Leveille SG, Markides KS, Ostir GV, Studenski S, Berkman LF, Wallace RB: Lower extremity function and subsequent disability: consistency across studies, predictive models, and value of gait speed alone compared with the short physical performance battery. J Gerontol A Biol Sci Med Sci 2000;55:M221-M231.

20 Walston J, Hadley EC, Ferrucci L, Guralnik JM, Newman AB, Studenski SA, Ershler WB, Harris T, Fried LP: Research agenda for frailty in older adults: toward a better understanding of physiology and etiology. Summary from the American Geriatrics Society/National Institute on Aging Research Conference on Frailty in Older Adults. J Am Geriatr Soc 2006;54:991-1001.

21 Unsworth J, Mode A: Preventing falls in older people: risk factors and primary prevention through physical activity. Br J Community Nurs 2003;8:214-220.

-22 deRekeneire N, Visser M, Peila R, Nevitt MC, Cauley JA, Tylavsky FA, et al: Is a fall just a fall: correlates of falling in healthy older persons. The Health, Aging, and Body Composition study. J Am Geriatr Soc 2003;51:841846

23 McAuley E, Morris KS, Doerksen SE, Motl RW, Liang $H$, White SM, Wójcicki TR, Rosengren K: Effects of change in physical activity on physical function limitations in older women: mediating roles of physical function performance and self-efficacy. J Am Geriatr Soc 2007;55:1967-1973.

- 24 Rogers MW, Johnson ME, Martinez KM, Mille ML, Hedman LD: Step training improves the speed of voluntary step initiation in aging. J Gerontol A Biol Sci Med Sci 2003; 58:46-51.

25 Maki BE: Gait changes in older adults: predictors of falls or indicators of fear. J Am Geriatr Soc 1997;45:313-320. 